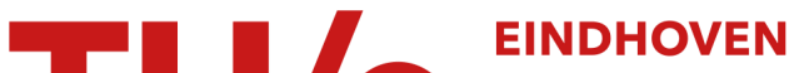 UNIVERSITY OF TECHNOLOGY
}

\section{Generalized behavior of the breakup of viscous drops in confinements}

Citation for published version (APA):

Janssen, P. J. A., Vananroye, A., Puyvelde, van, P. C. J., Moldenaers, P., \& Anderson, P. D. (2010). Generalized behavior of the breakup of viscous drops in confinements. Journal of Rheology, 54(5), 1047-1060. https://doi.org/10.1122/1.3473924

DOI:

10.1122/1.3473924

Document status and date:

Published: 01/01/2010

\section{Document Version:}

Publisher's PDF, also known as Version of Record (includes final page, issue and volume numbers)

\section{Please check the document version of this publication:}

- A submitted manuscript is the version of the article upon submission and before peer-review. There can be important differences between the submitted version and the official published version of record. People interested in the research are advised to contact the author for the final version of the publication, or visit the $\mathrm{DOI}$ to the publisher's website.

- The final author version and the galley proof are versions of the publication after peer review.

- The final published version features the final layout of the paper including the volume, issue and page numbers.

Link to publication

\section{General rights}

Copyright and moral rights for the publications made accessible in the public portal are retained by the authors and/or other copyright owners and it is a condition of accessing publications that users recognise and abide by the legal requirements associated with these rights.

- Users may download and print one copy of any publication from the public portal for the purpose of private study or research.

- You may not further distribute the material or use it for any profit-making activity or commercial gain

- You may freely distribute the URL identifying the publication in the public portal.

If the publication is distributed under the terms of Article 25fa of the Dutch Copyright Act, indicated by the "Taverne" license above, please follow below link for the End User Agreement:

www.tue.nl/taverne

Take down policy

If you believe that this document breaches copyright please contact us at:

openaccess@tue.nl

providing details and we will investigate your claim. 


\title{
Generalized behavior of the breakup of viscous drops in confinements
}

\author{
P. J. A. Janssen \\ Materials Technology, Eindhoven University of Technology, \\ P.O. Box 513, 5600 MB Eindhoven, The Netherlands \\ A. Vananroye, P. Van Puyvelde, and P. Moldenaers \\ Department of Chemical Engineering, Leuven Materials Research Centre, \\ K.U. Leuven, W. de Croylaan 46, B-3001 Leuven, Belgium \\ P. D. Anderson ${ }^{\text {a) }}$ \\ Department of Materials Technology, Eindhoven University of Technology, \\ P.O. Box 513, 5600 MB Eindhoven, The Netherlands
}

(Received 2 December 2009; final revision received 13 May 2010; published 25 August 2010)

\begin{abstract}
Synopsis
The breakup of confined drops in shear flow between parallel plates is investigated as a function of viscosity ratio and confinement ratio. Using a boundary-integral method for numerical simulations and a counter-rotating experimental device, critical capillary numbers in shear flow are obtained. It is observed that different viscosity ratios yield different trends with increasing confinement ratio: a low viscosity ratio drop shows an increase in critical capillary number, at a viscosity ratio of unity no major trend is seen, and the critical capillary number for a high viscosity ratio drop decreases significantly. A generalized explanation for all viscosity ratios is that confinement affects the orientation of the drop with respect to the direction of the local strain field. At moderate confinement ratios, the drop orients more toward the strain direction, where it experiences a stronger flow and hence, the critical capillary number is decreased. As the drop gets more confined, it aligns more in the flow direction. Hence, the drop experiences a weaker flow and thus, additionally stabilized by wall effects, it breaks at a higher critical capillary number. In principle, this behavior is the same for all viscosity ratios, but transitions occur at different confinement ratios. Most of the breakup is of a binary nature, but ternary breakup can occur if the drop length is larger than 6 undeformed drop radii, consistent with arguments based on the Rayleigh-Plateau instability. (C) 2010 The Society of Rheology. [DOI: 10.1122/1.3473924]
\end{abstract}

\section{INTRODUCTION}

Breakup of drops is, together with coalescence, the most important mechanism in the morphology development of blends [Tucker and Moldenaers (2002)]. Assuming two

\footnotetext{
a) Author to whom correspondence should be addressed; electronic mail: p.d.anderson@tue.nl
} 
non-mixable fluids both with a Newtonian viscosity and an interfacial tension $\sigma$ acting between the drop and matrix phase, the two main parameters that determine the breakup behavior are the capillary number $\mathrm{Ca}$ and the viscosity ratio $\lambda$. The capillary number is defined as the ratio between the viscous stresses distorting, and the interfacial stresses restoring the drop shape: $\mathrm{Ca}=\mu_{0} R \dot{\gamma} / \sigma$, with $\mu_{0}$ the viscosity of the matrix fluid, $R$ the radius of the undeformed drop, and $\dot{\gamma}$ the shear rate. The viscosity ratio is the ratio of the drop viscosity to the matrix viscosity: $\lambda=\mu_{1} / \mu_{0}$ with $\mu_{1}$ the viscosity of the drop, or dispersed phase. Depending on the flow type and viscosity ratio, a critical capillary number $\mathrm{Ca}_{\text {crit }}$ exists above which the drop breaks up.

Ever since the pioneering work of Taylor $(1932,1934)$, numerous studies have been conducted to investigate the deformation of drops up to and including breakup, which have been reviewed multiple times [Rallison (1984); Stone (1994); Tucker and Moldenaers (2002); Cristini and Renardy (2006)]. Of particular note is a systematic study in shear flow of drop breakup as a function of viscosity ratio, conducted by Grace (1982). This data set is now known as the Grace curve where, for shear flow, a minimum in critical capillary number of around 0.4 is found for a viscosity ratio of 0.6 . High viscosity ratio drops $(\lambda>4)$ are impossible to break in simple shear flow, as these drops tumble in the flow field, eventually reaching a steady state almost perfectly aligned with the flow direction. Low viscosity ratio drops asymptotically approach a critical capillary number of $\infty$ with decreasing viscosity ratio, scaling as $\mathrm{Ca}_{\text {crit }} \sim \lambda^{-2 / 3}$ according to a slender-body theory [Hinch and Acrivos (1980)]. A fit describing the Grace curve was made by de Bruijn (1989). Using a four-roll mill, Bentley and Leal (1986) generated Grace-like graphs for flow types ranging from simple shear to extensional flow. The critical capillary number was observed to decrease for all viscosity ratios, when the flow type changed from shear to extensional flow.

Recently, microfluidic devices have been given more attention [Stone et al. (2004); Cristini and Tan (2004); Van Puyvelde et al. (2008)]. There, a third parameter besides the capillary number and the viscosity ratio has to be taken into account: the effect of the confinement ratio, defined as the ratio between the drop diameter $2 R$ and the wall separation $2 W$. Investigating the breakup behavior of drops in shear flow, Vananroye et al. (2006) found that confined high viscosity ratio drops are easier to break, and confined low viscosity ratio drops are harder to break. This difference in behavior has remained a bit of a puzzle up until now, and is the focus of this paper.

Minale (2008) recently extended the Maffettone-Minale model [Maffettone and Minale (1998)] to include the Shapira-Haber result [Shapira and Haber (1990)], which in turn is an extension of Taylor's small deformation theory, to describe confined drops. Despite the limitation that the model can only describe an ellipsoidal droplet shape, it predicted an increase in $\mathrm{Ca}_{\text {crit }}$ with increasing confinement ratio for $\lambda<1$, minor effects for $\lambda=1$, and a drop in $\mathrm{Ca}_{\text {crit }}$ with increasing confinement ratio for $\lambda>1$. The results, however, did not line up accurately with experiments, and did not provide a physical explanation of the observed phenomena. Although the same trends were observed, the behavior was quantitatively different, as for example deviations from the unconfined case were only seen at the highest confinement ratios.

Recently, a modified version of the Hinch and Acrivos slender-body theory [Hinch and Acrivos (1980)] for low viscosity ratio drops in shear flow has been proposed [Janssen et al. (2010)], taking into account confinement effects. The main result of this analysis is that an extremely confined drop aligns more in the flow direction and becomes shorter, which leads to an increase of the critical capillary number with a factor of $\sqrt{3}$. The model, however, does not describe intermediate confinement ratios.

The objective of this paper is to investigate the dependency of the critical capillary 


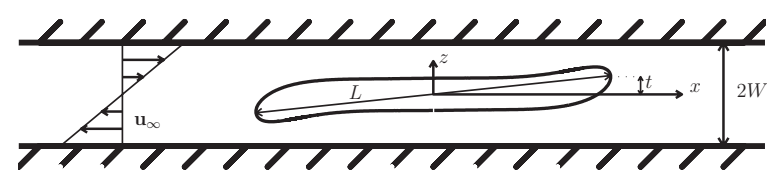

FIG. 1. Schematic representation of a highly deformed drop in a matrix fluid between two parallel plates located at $z= \pm W$. Also shown are the definitions of the drop length $L$, the tip deflection $t$, and the prescribed shear flow $\mathbf{u}_{\infty}$.

number on the viscosity and the confinement ratio in detail, covering a wide span of viscosity ratios and confinement ratios, and to explain the observed differences between the viscosity ratios. Experimental and numerical techniques will be used complementarily to each other: an advantage of experiments is the ability to easily probe the low viscosity ratio regime, whereas simulations provide easier access to quantities such as drop length and orientation. The results from both methods overlap in a large window of viscosity ratios. In Sec. II, the numerical procedure and the experimental methods and materials are outlined, after which the results are shown in Sec. III. Section IV contains an explanation and discussion for the differences in behavior between the viscosity ratios, and a criterion for ternary breakup. Final conclusions are drawn in Sec. V.

\section{METHODS}

\section{A. Numerical procedure}

Consider a Newtonian drop with an undeformed radius $R$ in creeping flow conditions in a Newtonian matrix between parallel walls, with the walls located at $z= \pm W$. Due to the shear field, indicated by $\mathbf{u}_{\infty}$, the drop will deform and orient, which is generally expressed by means of the drop major axis $L$ and the orientation angle $\theta$. However, we use the tip deflection $t$ (schematically shown in Fig. 1) instead of $\theta$ to quantify orientation, as motivated later in the paper. To simulate the deformation of a drop, a recently developed 3D boundary-integral method is used which takes into account the presence of the walls [Janssen and Anderson $(2007,2008)]$. In the numerical procedure, all length scales are scaled with $R$, time with $\dot{\gamma}$, velocities with $R \dot{\gamma}$ and pressures with $\sigma / R$. Due to this scaling, the two parameters that characterize the flow problem, besides $\lambda$, are the confinement ratio $R / W$, and the capillary number $\mathrm{Ca}=R \dot{\gamma} \mu_{0} / \sigma$.

The boundary-integral method gives the velocity $\mathbf{u}$ at the pole $\mathbf{x}_{0}=\left(x_{0}, y_{0}, z_{0}\right)^{T}$ by

$$
(\lambda+1) \mathbf{u}\left(\mathbf{x}_{0}\right)=2 \mathbf{u}_{\infty}\left(\mathbf{x}_{0}\right)-\frac{1}{4 \pi} \int_{S} \mathbf{f}(x) \cdot \mathbf{G}\left(\mathbf{x}, \mathbf{x}_{0}\right) \mathrm{d} S(\mathbf{x})-\frac{\lambda-1}{4 \pi} \int_{S} \mathbf{u}(x) \cdot \mathbf{T}\left(\mathbf{x}, \mathbf{x}_{0}\right) \cdot \mathbf{n}(x) \mathrm{d} S(\mathbf{x}),
$$

where the integration is over the drop surface $S$. The discontinuity in the normal stress across the interface is given by $\mathbf{f}$, which reads in nondimensional form:

$$
\mathbf{f}(\mathbf{x})=\frac{2}{\mathrm{Ca}} \kappa(\mathbf{x}) \mathbf{n}(\mathbf{x})
$$

with $\mathbf{n}$ the vector normal to the interface and $\kappa$ the local mean curvature.

The requirement that the velocity components should vanish at the wall is obeyed by modifying the Green's functions $\mathbf{G}$ and $\mathbf{T}$ to include the free-space result and a part with the additional contributions due to the presence of the walls: 
TABLE I. Zero-shear viscosities at $24{ }^{\circ} \mathrm{C}$ and activation energies of the blend components; viscosity ratios of the blends at $24^{\circ} \mathrm{C}$.

\begin{tabular}{lccc}
\hline \hline Grade & & & \\
\hline$\eta_{0}\left(24{ }^{\circ} \mathrm{C}\right)$ & $\begin{array}{c}E_{a} \\
(\mathrm{~Pa} \mathrm{~s})\end{array}$ & $\begin{array}{c}\lambda=\frac{\eta_{\mathrm{PDMS}}}{\eta_{\mathrm{PIB}}} \\
(\mathrm{kJ} / \mathrm{mole})\end{array}$ & $\left(24{ }^{\circ} \mathrm{C}\right)$ \\
\hline PIB Parapol 1300 & 101 & 64.4 & Matrix \\
PDMS 200 ${ }^{\circledR}$ fluid & 0.99 & 12.6 & 0.01 \\
PDMS Rhodorsil 47V12500 & 12.2 & 12.6 & 0.12 \\
PDMS Silbione 70047V30000 & 30 & 12.6 & 0.30 \\
PDMS Rhodorsil 47V100000 & 103 & 12.9 & 1.02 \\
PDMS Rhodorsil 47V200000 & 200 & 12.6 & 1.98 \\
PDMS Rhodorsil 47V500000 & 493 & 12.5 & 4.88 \\
PDMS Rhodorsil 47V1000000 & 1142 & 12.7 & 11.3 \\
\hline \hline
\end{tabular}

$$
\mathbf{G}=\mathbf{G}^{\infty}+\mathbf{G}^{2 W}, \quad \mathbf{T}=\mathbf{T}^{\infty}+\mathbf{T}^{2 W},
$$

where the free-space parts are given by

$$
\mathbf{G}^{\infty}\left(\mathbf{x}, \mathbf{x}_{\mathbf{0}}\right)=\frac{\mathbf{I}}{|\hat{\mathbf{x}}|}+\frac{\hat{\mathbf{x}} \hat{\mathbf{x}}}{|\hat{\mathbf{x}}|^{3}}, \quad \mathbf{T}^{\infty}\left(\mathbf{x}, \mathbf{x}_{\mathbf{0}}\right)=-6 \frac{\hat{\mathbf{x}} \hat{\mathbf{x}} \hat{\mathbf{x}}}{|\hat{\mathbf{x}}|^{5}},
$$

with $\hat{\mathbf{x}}=\mathbf{x}-\mathbf{x}_{0}$ and $\mathbf{x}=(x, y, z)^{T}$ the field point. Definitions of the wall modifications are derived by Jones (2004). Fast and accurate computation of these wall-modified Green's functions is done by subtracting slow-decaying terms [Staben et al. (2003)]. More details for the current implementation are worked out in Janssen and Anderson (2007, 2008).

Furthermore, a remesh algorithm is employed to handle large deformations [Cristini et al. $(1998,2001)]$. A non-singular contour integration is applied to handle the singularity of the free-space Green's functions at the drop interface, and a multi-time step scheme to limit the number of Green's functions that have to be computed [Bazhlekov et al. (2004)]. A comparison of this model with experimental data was performed by Vananroye et al. (2008b) for a viscosity ratio of unity. It was shown that the numerical model is well capable of predicting the shape, deformation, orientation, and breakup of confined drops in shear flow.

\section{B. Experimental procedure}

\section{Materials}

The fluids used in the present work are a polyisobutylene (PIB) grade as the matrix phase (Parapol, obtained from ExxonMobil Chemical, Houston, TX) and several grades of polydimethyl siloxane (PDMS) as the drop phase (Rhodorsil and Silbione, obtained from Rhodia Chemicals, France). These transparent materials all have a constant viscosity under the experimental flow conditions, and since elasticity effects can be neglected, they are considered to behave as Newtonian liquids [Vinckier et al. (1996)]. In addition, the densities of the pure materials are nearly matching $\left(\rho_{\text {PIB }}=890 \mathrm{~kg} / \mathrm{m}^{3}\right.$ at $20{ }^{\circ} \mathrm{C}$ and $\rho_{\text {PDMS }}=970 \mathrm{~kg} / \mathrm{m}^{3}$ at $20^{\circ} \mathrm{C}$ ) [Minale et al. (1997)]. Table I presents the measured zeroshear viscosities $\eta_{0}$ at $24{ }^{\circ} \mathrm{C}$ (ARES-LS from TA Instruments) and the activation energies $E_{a}$ of the components. Furthermore, the viscosity ratios $\lambda$ of the several PDMS/PIB systems are summarized. The interfacial tension $\sigma$ of these systems, which was measured 
to be $2.8 \mathrm{mN} / \mathrm{m}$ [Sigillo et al. (1997)], is independent of the molecular weight of PDMS since only grades with relatively high molecular weights are used here [Kobayashi and Owen (1995)].

\section{Methods}

Breakup studies are performed in a counter-rotating parallel plate flow cell (PaarPhysica) which has been described in detail in Vananroye et al. (2006). The device consists of two glass plates controlled by separate motors. This way, in counter-rotating mode, a stagnation plane is created during flow, which facilitates microscopic observations. The entire setup is located in a thermostated room in which the temperature is carefully monitored during the experiments. The matrix material is loaded in a glass cup surrounding the bottom plate, and the gap is fixed at $1 \mathrm{~mm}$. The degree of confinement is varied by injecting drops with different sizes (diameter $2 R$ ranging from 100 to $900 \mu \mathrm{m}$ ) in the matrix fluid at radial positions far enough from the rotation center to ensure a uniform shear field. During deformation and breakup, a drop is observed by means of a Wild M5A stereo microscope and a Basler A301f camera in both the vorticity ( $y$ direction in Fig. 1), as well as the velocity gradient direction ( $z$ direction). The Reynolds number, which can be written as $\mathrm{Re}=\mathrm{Ca} 2 \rho \sigma \mathrm{W} / \mu_{0}^{2}$, is in the order of $10^{-6}$ which justifies the creeping flow assumption.

\section{Definitions}

\section{Critical capillary number}

The critical capillary number is defined in this study as the lowest capillary number found at which an initially spherical drop breaks up. Alternative definitions are complicated or require time-consuming procedures in both experiments and numerical simulations. Complications include the significant influence of the deformation history on the value of $\mathrm{Ca}_{\text {crit }}$ [Torza et al. (1972); Tucker and Moldenaers (2002)], slow dynamics and overshoots near critical situations [Janssen and Anderson (2007)], and multiple stationary drop shapes near the critical capillary number [Bławzdziewicz et al. (2002, 2003); Young et al. (2008)]. Additional problems to overcome in long experiments are the drift of the drop out of the camera view, a small offset of the drop's mass center out of the center plane between the walls, and diffusion of small molecules which influences the interfacial tension [Guido et al. (1999); Shi et al. (2004); Tufano et al. (2008, 2010)].

\section{Protocol}

As mentioned above, in all cases discussed here, a spherical drop is initially placed halfway between the walls. An experiment is started with a low capillary number at which the drop does not break up. Then, when the steady state is reached, the experiment is interrupted and after retraction of the drop, flow is restarted at a slightly higher capillary number (increase of 0.02 or lower). This procedure is repeated until breakup occurs. A similar procedure is used for the simulations, with the option to run multiple simulations in parallel. Typically a range of capillary numbers is simulated near the expected $\mathrm{Ca}_{\text {crit }}$ based on either experimental data or lower confinement ratios. In a second run, the capillary number is further refined until the difference between the highest capillary number that leads to a steady state, and the lowest capillary number that leads to breakup is small enough (in most cases 0.01). 


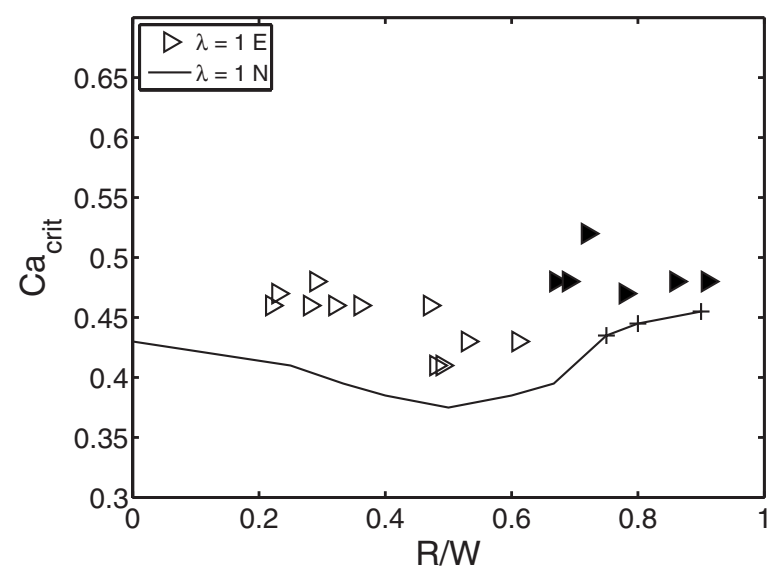

FIG. 2. $\mathrm{Ca}_{\text {crit }}$ as a function of confinement ratio $R / W$ for unit viscosity ratio drops. Symbols represent experimental data $(E)$, while the full line represents the numerical results $(\mathrm{N})$. Ternary breakup is indicated with filled symbols for the experiments and with + for the simulations.

\section{Ternary breakup}

We define ternary breakup as a situation in which the drop breaks up into three or more, more or less equal-sized parts, contrary to binary breakup where the drop breaks in two parts, with or without much smaller satellite drops. Although the simulations are perfectly symmetric, in most experimental situations one side of the drop has the tendency to break off earlier due to the fact that the drop is not always positioned at the centerline between the plates. This is still referred to as ternary breakup.

\section{Drop alignment}

To quantify the alignment of the drop in the flow direction, we use the tip deflection, as illustrated in Fig. 1. The orientation angle $\theta$, which is normally used, did not provide clearly distinguishable results between different situations. As long as the drop is ellipsoidal, the orientation angle is a well defined and intuitive parameter. However, the drop shapes presented here, which are close to breakup, are far from ellipsoidal (see Fig. 1). In addition, the drop length increases significantly with increasing confinement ratio, automatically leading to a decrease in the orientation angle, which makes a quantitative comparison between the results difficult. As will be shown, the tip deflection gave more satisfying results.

\section{EFFECT OF VISCOSITY RATIO AND CONFINEMENT ON $\mathrm{Ca}_{\text {crit }}$}

\section{A. Unit viscosity ratio drops}

In Fig. 2, numerical and experimental results for the critical capillary number as a function of the confinement ratio are shown for a viscosity ratio of unity [see also Janssen and Anderson (2007) and Vananroye et al. (2006)]. Good agreement is obtained between the experimental and numerical data. Due to an unidentified systematic error, we found in all data sets that the experiments gave a slightly higher critical capillary number, but trends were found to be identical. For low confinement ratios, the bulk flow result is recovered $\left(\mathrm{Ca}_{\text {crit }}=0.43\right)$. Increasing the confinement ratio leads to a small decrease in the critical capillary number until a minimum in $\mathrm{Ca}_{\text {crit }}$ is reached at a confinement ratio of approximately 0.5 . Further increase in $R / W$ leads to a small increase of the critical 


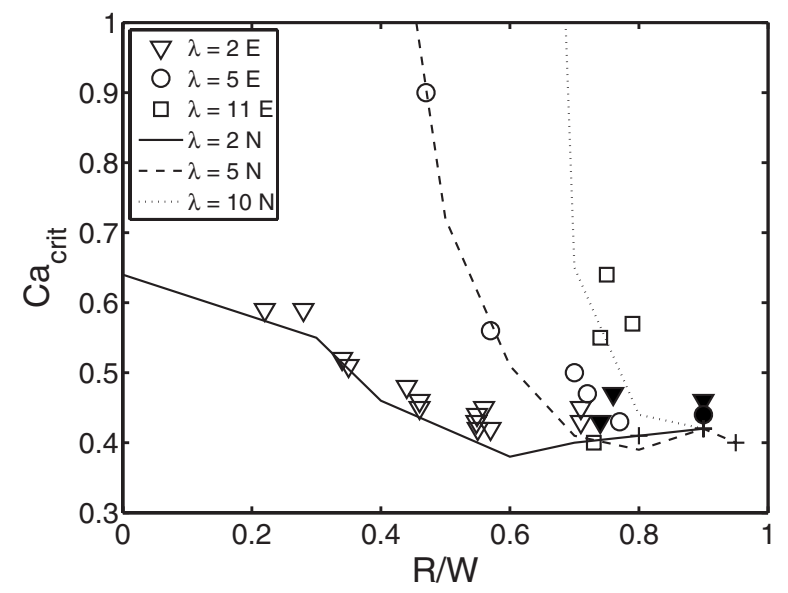

FIG. 3. $\mathrm{Ca}_{\text {crit }}$ as a function of confinement ratio $R / W$ for high viscosity ratio drops. Symbols represent experimental data $(\mathrm{E})$, while lines represent the numerical results $(\mathrm{N})$. Ternary breakup is indicated with filled symbols for the experiments and with + for the simulations.

capillary number. Contrary to unconfined flow, where binary breakup occurs, we observe ternary breakup (indicated by the filled symbols in the experimental graphs and + symbols for the numerical data) above a confinement ratio of approximately 0.7. In Figs. 5(a) and 5(b), representative drop shapes are shown resulting from simulations and experiments, respectively.

\section{B. High viscosity ratio drops}

Numerical and experimental results for high viscosity ratio drops are shown in Fig. 3. Again, the similarities in the experimental and numerical data are striking. A massive decrease in critical capillary number is observed with increasing confinement ratio, especially for the highest viscosity ratios [Vananroye et al. (2006)]. Both simulations and experiments report breakup for viscosity ratios as high as $\lambda=10$ at high confinement ratios, a phenomenon that does not occur in bulk shear flow. Ternary breakup is observed, although only for $R / W \geq 0.8$, which is at higher confinement ratios than for a viscosity ratio of unity $(R / W>0.7)$. For $\lambda=10$, ternary breakup is not even observed at the highest confinement ratio investigated here. Between $R / W=0.8$ and $R / W=0.9$, the critical capillary number marginally increases for $\lambda=2$ and 5 . We also note that the lowest critical capillary number for all viscosity ratios seems to be about 0.4 . For $\lambda=5$, several numerically and experimentally obtained drop shapes at breakup are shown in Figs. 5(a) and $5(\mathrm{~b})$, respectively.

\section{Low viscosity ratio drops}

Critical capillary numbers for breakup of low viscosity ratio drops are shown in Fig. 4. Although there is only limited data from the simulations $(\lambda=0.3)$, the match with the experiments is excellent. Contrary to the high viscosity ratio case (Fig. 3), we now see a relatively large increase of the critical capillary number with increasing confinement ratio. Also, ternary breakup occurs here at rather low confinement ratios. For low viscosity ratios, the slender-body theory predicts a critical capillary number for confined drops that is $\sqrt{3}$ times higher than the unconfined case [Janssen et al. (2010)]. Although this limit is not reached in the simulations because boundary integral simulations for small $\lambda$ 


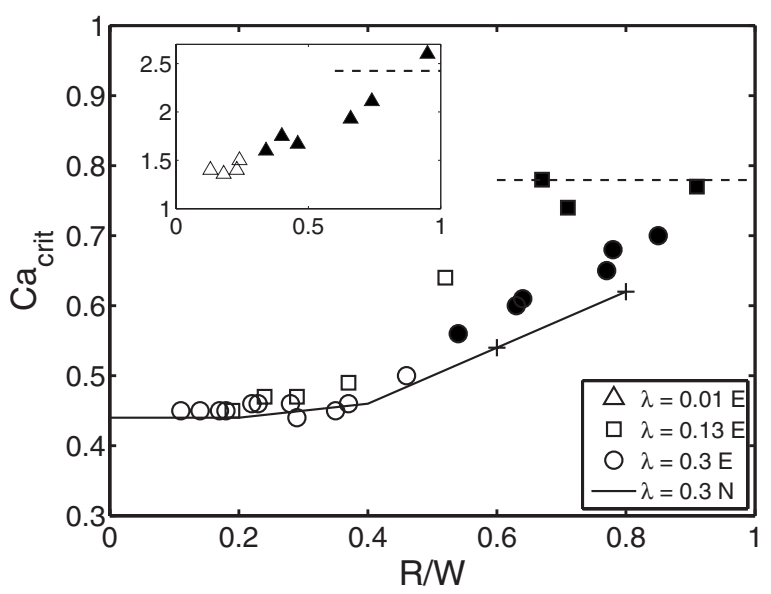

FIG. 4. $\mathrm{Ca}_{\text {crit }}$ as a function of confinement ratio $R / W$ for low viscosity ratio drops. Symbols represent experimental data (E), while the line represents the numerical results at $\lambda=0.3(\mathrm{~N})$. Ternary breakup is indicated with filled symbols for the experiments and with + for the simulations. The dashed line represents the limiting critical capillary number that is $\sqrt{3}$ times the unconfined one, based on the experimental data [Janssen et al. (2010)].

are extremely time consuming, the critical capillary number at the highest experimental confinement ratios hovers around this ratio. However, with the uncertainty on the data, it is impossible to judge whether a limit is truly reached and whether the limiting ratio is exactly $\sqrt{3}$. Drop shapes are shown in Figs. 5(a) and 5(b) for simulations and experiments, respectively, at $\lambda=0.3$.

\section{Drop length and orientation}

In addition to the critical capillary number, the numerically obtained dimensionless drop length $L$ and tip deflection $t$ of drops at the highest sub-critical capillary number are shown in Figs. 6(a) and 6(b), respectively, for several viscosity ratios. For the drop length $L$, a relatively constant value is observed at low confinement ratios. An increase in $L$ is seen for all viscosity ratios at confinement ratios exceeding the one at which the minimal

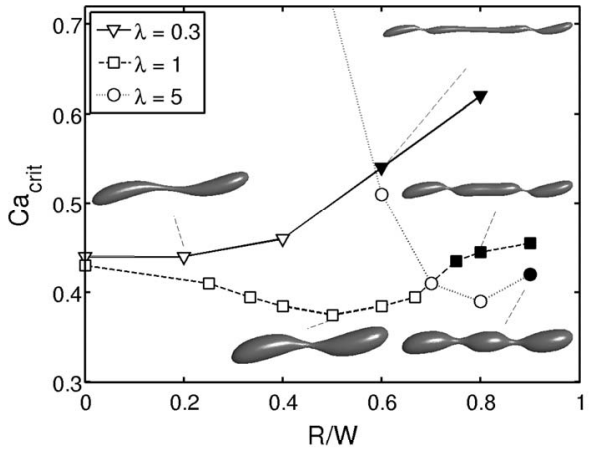

(a)

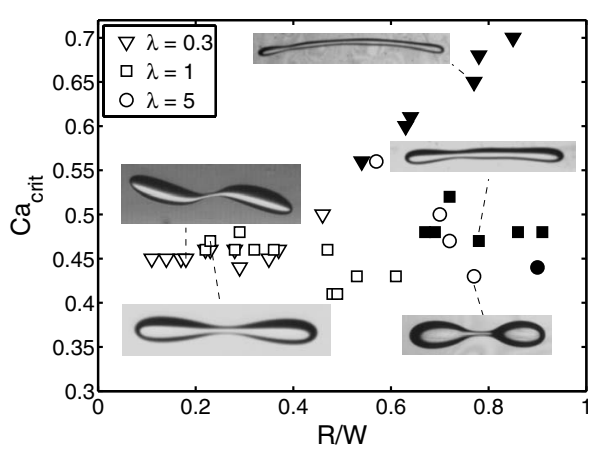

(b)

FIG. 5. $\mathrm{Ca}_{\text {crit }}$ from (a) simulations and (b) experiments as a function of the confinement ratio $R / W$ for $\lambda$ $=0.3,1$, and 5 , with several drop shapes just before breakup. Filled symbols indicate ternary breakup. 


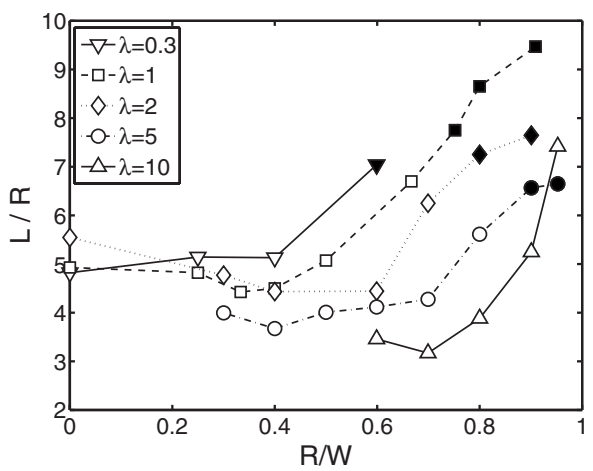

(a)

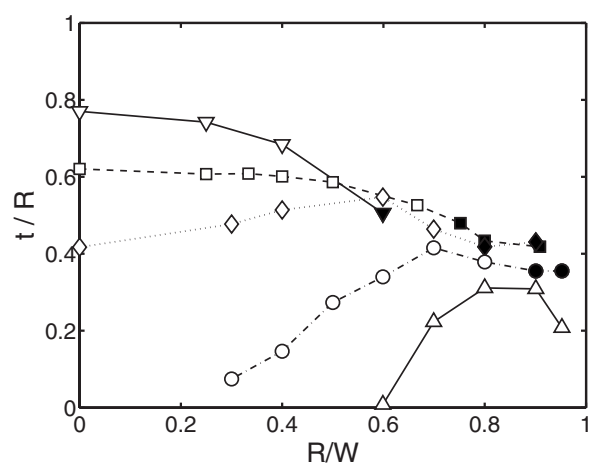

(b)

FIG. 6. Numerical results for (a) the dimensionless drop length $L$ and (b) the dimensionless tip deflection $t$ at the highest sub-critical capillary number as a function of the confinement ratio $R / W$ for various viscosity ratios $\lambda$. Filled symbols indicate ternary breakup.

$\mathrm{Ca}_{\text {crit }}$ is found. This trend is seen for all viscosity ratios. The tip deflection shows an increase, followed by a decrease, with the maximum deflection found at the confinement ratio corresponding to the minimal value of $\mathrm{Ca}_{\text {crit }}$.

\section{MECHANISM FOR DROP BREAKUP IN CONFINEMENT}

\section{A. Generalized behavior}

In Sec. III, it was shown both experimentally and numerically that confinement causes an increase in $\mathrm{Ca}_{\text {crit }}$ at low viscosity ratios, at $\lambda=1$ no major trend is seen, and $\mathrm{Ca}_{\text {crit }}$ at high viscosity ratios is lowered significantly by confinement. In addition, we showed the trends for the drop length and orientation, expressed by the tip deflection.

A generalized explanation, describing the behavior at all viscosity ratios in a uniform way, is schematically shown in Fig. 7 where the critical capillary number as function of the confinement ratio is categorized in five regions. The main effect of confinement is a change in the orientation of the drop with respect to the direction of the strain field, which is oriented under an angle of $45^{\circ}$ with the velocity direction in bulk shear flow.

In region $\mathrm{I}$, the effect of the confinement is insignificant (typically $R / W<0.3$ ), the unconfined behavior is found, and the original Grace curve is recovered [Grace (1982)].

In region II, at moderate degrees of confinement, the effect of confinement at subcritical steady-state conditions is a suppression of the rotation of the drop toward the flow direction: the tip deflection becomes larger. Thus, the drop, which is more aligned in the straining direction of the flow, experiences a stronger flow and the steady-state drop deformation increases with increasing degree of confinement at a fixed capillary number $\mathrm{Ca}$. Hence, the confined drop reaches the critical breakup length at a lower capillary number than the unconfined one, and breakup occurs at lower $\mathrm{Ca}$. The higher the confinement ratio, the larger the decrease in $\mathrm{Ca}_{\text {crit }}$. We do not expect that the walls have a significant stabilizing effect at this confinement ratio, and hence the critical breakup length $L_{\text {crit }}$ is the same for confined and unconfined droplets in this region, also supported by the data in Fig. 6. 


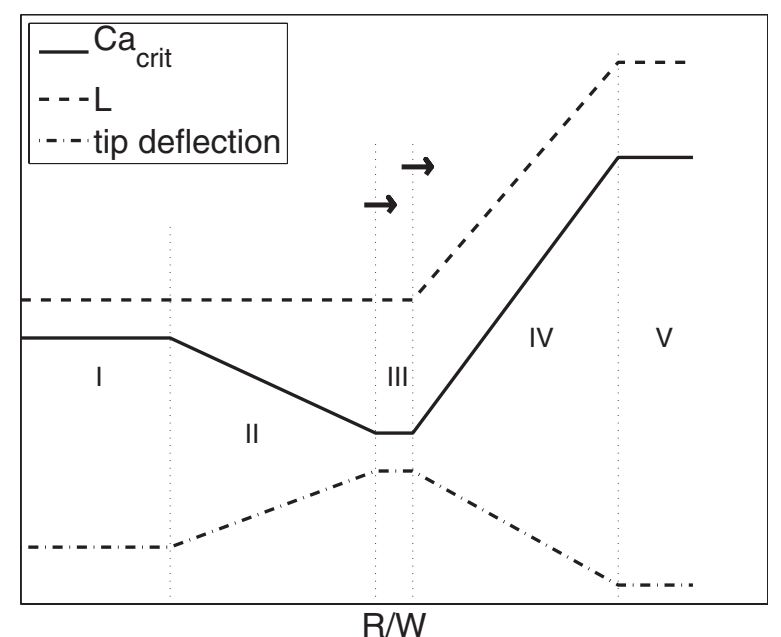

FIG. 7. Schematic representation of the effect of the confinement ratio $R / W$ on $\mathrm{Ca}_{\text {crit }}$. The five regions (I-V) are explained in the text. Trends for the drop length $L$ and tip deflection $t$ at the highest sub-critical $\mathrm{Ca}$ are shown. Transitions are shifted to higher confinement ratios with increasing viscosity ratio, as indicated by the arrows.

In region III, some sort of equilibrium between regions II and IV is reached. Here, the drop reaches its maximum orientation toward the direction of the strain field, which results in a minimum in $\mathrm{Ca}_{\text {crit }}$ as a function of confinement ratio, as seen in Fig. 7, as well as a maximum in the tip deflection.

With increasing degree of confinement, region IV is entered. Due to the large confinement ratio, the deformed drop cannot maintain its orientation toward the strain field during flow, as its orientation is limited by the presence of the walls. Hence, the drop rotates away from the strain direction, indicated by a lower tip deflection. This leads to a weaker flow and the critical breakup length $L_{\text {crit }}$ is reached at higher capillary numbers: $\mathrm{Ca}_{\text {crit }}$ goes up. In addition, due to a stabilizing effect of the walls, $L_{\text {crit }}$ also increases, and therefore $\mathrm{Ca}_{\text {crit }}$ increases even more.

A long drop generated at sub-critical capillary numbers in region IV is typically not stable in bulk conditions, but due to the stabilizing effect of the walls and the weak orientation of the drop relative to the direction of the strain field, this steady-state elongated shape can be obtained under confinement [Migler (2001)]. The long drop eventually breaks up in three or more equally sized drops (ternary breakup). In this case, effects similar to Rayleigh disturbances start to play a role [Lord Rayleigh (1879)]. The exact details of the breakup mechanism are beyond the scope of this work, but it is worthy to note that ternary breakup usually occurs as the drop is retracting from the overshoot [see, for example, transient data in Sibillo et al. (2006), Janssen and Anderson (2007), and Vananroye et al. (2008a)]. In Sec. IV B, a simple scaling argument is presented for the minimal dimensionless drop length required to have ternary breakup. Torza et al. (1972) showed that by increasing the shear rate from a sub-critical level to a value well above the critical one, they could generate large satellite drops, which could also be considered ternary breakup. In principle, this is a situation where a step in shear rates is made to a capillary number that is much larger than $\mathrm{Ca}_{\text {crit }}$. A more systematic experimental characterization of breakup at high capillary numbers was conducted by Zhao (2007).

Region $\mathrm{V}$ is a hypothetical situation, since it is very difficult to reach experimentally as well as computationally. It is included to indicate that the alignment and stretching of 
a drop cannot continue indefinitely, since other physical effects start to play a role. We expect that the asymptotic region found by Janssen et al. (2010) should be reached here, where $\mathrm{Ca}_{\text {crit }}$ is $\sqrt{3}$ times the unconfined $\mathrm{Ca}_{\text {crit }}$. This theory, however, is only defined for low viscosity ratios and does not consider additional stabilizing effects of the walls. It is therefore unknown for which range of viscosity ratios and confinement ratios the asymptotic theory is valid.

It is now postulated that all viscosity ratios show the same behavior for these five regions, except that the viscosity ratio introduces a shift over the horizontal confinement ratio axis, indicated by the arrows in Fig. 7.

As was shown in Fig. 3, a moderately confined drop with a high viscosity ratio (especially $\lambda=5$ and $\lambda=10$ ) initially shows a strong decrease in $\mathrm{Ca}_{\text {crit }}$, corresponding to region II in Fig. 7. Also the critical breakup length and tip deflection presented in Fig. 6 for high viscosity ratios initially follow the trends suggested in region II in Fig. 7. For these viscosity ratios, a small increase in $\mathrm{Ca}_{\text {crit }}$ (region IV) is seen at very high confinement ratios where the critical tip deflection indeed decreases and the critical length increases, as seen in Fig. 6. Remarkably, the minimum $\mathrm{Ca}_{\text {crit }}$ is again about 0.4 for $\lambda$ $=2$ and $\lambda=5$. In the data shown in Figs. 3 and 5(a), the high viscosity ratio drops indeed seem to show an increase in $\mathrm{Ca}_{\text {crit }}$, but only at the highest confinement ratios under investigation $(R / W=0.9$ and 0.95$)$, and this increase in $\mathrm{Ca}_{\text {crit }}$ is virtually non-existent at $\lambda=10$. It is not entirely known at this point what the behavior of an over-confined drop is $(R / W>1)$, and whether it is significantly different compared to more moderate confinement ratios. Sibillo et al. (2006) presented one result for $R / W=2$ with $\lambda=1$, which showed complicated, multi-stage breakup behavior. Examining the images, one could argue that this is also ternary breakup, with the middle drop in the second stage also breaking up into three smaller drops.

A low viscosity ratio drop, on the other hand (see Fig. 4), only shows an increase in $\mathrm{Ca}_{\text {crit }}$ with increasing degree of confinement. Hence, under these conditions of viscosity ratio, only regions IV and V are entered. The data in Fig. 6 confirm that above the critical confinement ratio $(R / W>0.3)$, a low viscosity ratio drop shows an increase in critical drop length, and a decrease in tip deflection, as was suggested for region IV.

At intermediate viscosity ratios (see, e.g., Fig. 2), all trends suggested in Fig. 7 are present. At moderate confinement ratios $(0.3<R / W<0.5) \mathrm{Ca}_{\text {crit }}$ slightly decreases, as suggested in region II. The results in Fig. 6 also show a constant critical breakup length in this region. The suggested increase in tip deflection is also weakly present. Around $R / W=0.5$, region III is entered, where a minimum in $\mathrm{Ca}_{\text {crit }}$ of 0.4 as function of the degree of confinement is found. Finally, under more severe confined conditions, $\mathrm{Ca}_{\text {crit }}$ and $L_{\text {crit }}$ increase, while the tip deflection decreases, corresponding to the trends shown in region IV.

\section{B. Criterion for ternary breakup}

We now present a simple scaling argument for the minimal length of a drop to show ternary breakup. The data in Fig. 6(a) suggest a minimum length around 6 undeformed drop radii. Assume that the ternary breakup is a result of a wave forming on the drop surface, which has a wavelength $\omega$ large enough to grow [Lord Rayleigh (1879); Tomotika (1935)]. This implies that the wavelength has to be larger than the circumference of the cross section $2 \pi r$, where $r$ is the local radius. We also assume, in a crude approximation, that the drop is cylindrical, so that the volume $V$ is $\pi L r^{2}=(4 \pi / 3) R^{3}$. To obtain ternary breakup, we need to have at least two wavelengths $\omega$ over the drop length $L$. After rescaling $L$ and $r$ with $R$, we arrive at 


$$
L / R>\left(\frac{8 \pi}{\sqrt{3}}\right)^{2 / 3}=5.95
$$

which qualitatively agrees with the value of 6 suggested earlier. The drop length $L$ has to be at least larger than 6 undeformed drop radii $R$ for the possibility to have ternary breakup, but a drop length larger than 6 does not automatically lead to ternary breakup, which may be obvious from the drop lengths reported in Fig. 6(a), which are stable values for sub-critical situations. In this case, the shear flow is most likely stabilizing the elongated drop against growth of instabilities by convecting waves in opposite directions, and the walls might also have a stabilizing effect [Son et al. (2003)]. One other additional remark about the ternary breakup is that during transient behavior, a drop at the largest confinement ratio first shows an overshoot in $L$, then it partially retracts and the three blobs are formed, and finally, it breaks up. So the values for stable, sub-critical values are even lower than the maximum $L$ reached prior to breakup (typical overshoot is in the order of 20\%-30\%). This analysis ignored the fact that the wavelength with the largest growth speed is a function of the viscosity and confinement ratio [Tomotika (1935); Mikami and Mason (1975); Son et al. (2003)]. However, this is not relevant here since we only looked for the minimum wavelength required.

\section{CONCLUSIONS}

We have investigated droplet breakup in confined shear flow, both experimentally and numerically. In low viscosity ratio situations, it is more difficult to break a drop in confinement than in bulk due to the fact that the drop aligns more in the direction of flow and hence experiences a weaker flow. In addition, the walls stabilize the drop shape causing it to be more elongated at the moment of breakup. At high viscosity ratios, a confined drop is easier to break than an unconfined one, as the confined drop orients more in the direction of the strain field. Hence, it experiences a stronger flow and reaches the critical breakup length at a lower capillary number. When the drop and the matrix are equi-viscous, a combination of this behavior is seen: at intermediate confinement ratios, a small reduction in the critical capillary number is seen similar to a highly viscous drop, while at high confinement ratios, an increase in critical capillary number is observed, similar to a low viscosity ratio drop. This increase is actually also present for a high viscosity ratio drop but only at very large confinement ratios. We conclude that the breakup behavior of a confined drop is actually similar for all viscosity ratios, but the viscosity ratio shifts the behavior over the confinement axis and changes the magnitude of the effects. In all cases, the effect of confinement on the breakup depends on the orientation of the drop with respect to the local strain field and the stabilizing effects of the walls. This is schematically summarized in Fig. 7. In addition, ternary breakup could be observed for all viscosity ratios when the drop becomes longer than 6 undeformed drop radii, a value support by an argument based on the Rayleigh-Plateau instability, although this criterion by itself is not sufficient to ensure ternary breakup.

\section{ACKNOWLEDGMENTS}

The authors P.J. and P.A. acknowledge support by the Dutch Polymer Institute (Project No. 446). A.V., P.V.P., and P.M. acknowledge the Onderzoeksfonds KULeuven (Grant Nos. GOA 03/06 and GOA 09/002). A.V. is a post-doctoral fellow of the FWO Vlaanderen. 


\section{References}

Bazhlekov, I., P. D. Anderson, and H. E. H. Meijer, "Non-singular boundary-integral method for deformable drops in viscous flows," Phys. Fluids 16, 1064-1081 (2004).

Bentley, B. J., and L. G. Leal, "An experimental investigation of drop deformation and breakup in steady, two-dimensional linear flows," J. Fluid Mech. 167, 241-283 (1986).

Bławzdziewicz, J., V. Cristini, and M. Loewenberg, "Critical behavior of drops in linear flows. I. Phenomenological theory for drop dynamics near critical stationary states," Phys. Fluids 14, 2709-2718 (2002).

Bławzdziewicz, J., V. Cristini, and M. Loewenberg, "Multiple stationary states for deformable drops in linear Stokes flow," Phys. Fluids 15, L37-L40 (2003).

Cristini, V., J. Blawzdziewicz, and M. Loewenberg, "Drop breakup in three-dimensional viscous flows," Phys. Fluids 10, 1781-1783 (1998).

Cristini, V., J. Blawzdziewicz, and M. Loewenberg, "An adaptive mesh algorithm for evolving surfaces: Simulation of drop breakup and coalescence,” J. Comput. Phys. 168, 445-463 (2001).

Cristini, V., and Y. Renardy, "Scalings for drop sizes in shear-driven breakup: Non-microfluidic ways to monodisperse emulsions," Fluid Dyn. Mater. Process. 2, 77-93 (2006).

Cristini, V., and Y.-C. Tan, "Theory and numerical simulation of droplet dynamics in complex flows-A review," Lab Chip 4, 257-264 (2004).

de Bruijn, R. A., "Deformation and breakup of drops in simple shear flows," Ph.D. thesis, Eindhoven University of Technology, The Netherlands, 1989.

Grace, H. P., "Dispersion phenomena in high viscosity immiscible fluid systems and application of static mixers as dispersion devices in such systems," Chem. Eng. Commun. 14, 225-277 (1982).

Guido, S., M. Simeone, and M. Villone, "Diffusion effects on the interfacial tension of immiscible polymer blends," Rheol. Acta 38, 287-296, 1999.

Hinch, E. J. and A. Acrivos, "Long slender drops in a simple shear flow," J. Fluid Mech. 98, 305-328 (1980).

Janssen, P. J. A., and P. D. Anderson, "Boundary-integral method for drop deformation between parallel plates," Phys. Fluids 19, 043602 (2007).

Janssen, P. J. A., and P. D. Anderson, “A boundary-integral model for drop deformation between two parallel plates with non-unit viscosity ratio drops," J. Comput. Phys. 227, 8807-8819 (2008).

Janssen, P. J. A., P. D. Anderson, and M. Loewenberg, "A slender-body theory for low-viscosity drops between parallel walls," Phys. Fluids 22, 042002 (2010).

Jones, R. B., "Spherical particle in Poiseuille flow between planar walls," J. Chem. Phys. 121, 483-500 (2004).

Kobayashi, H., and M. Owen, "Surface properties of fluorosilicones," Trends Polym. Sci. 3, 330-335 (1995).

Lord Rayleigh, "On the capillary phenomena of jets," Proc. R. Soc. London 29, 71-97 (1879).

Maffettone, P.-L., and M. Minale, "Equation of change for ellipsoidal drops in viscous flow," J. Non-Newtonian Fluid Mech. 78, 227-241 (1998).

Migler, K. B., "String formation in sheared polymer blends: Coalescence, breakup, and finite size effects," Phys. Rev. Lett. 86, 1023-1026 (2001).

Mikami, T., and S. G. Mason, "The capillary break-up of a binary liquid column inside a tube," Can. J. Chem. Eng. 53, 372-377 (1975).

Minale, M., "A phenomenological model for wall effects on the deformation of an ellipsoidal drop in viscous flow," Rheol. Acta 47, 667-675 (2008).

Minale, M., P. Moldenaers, and J. Mewis, "Effect of shear history on the morphology of immiscible polymer blends," Macromolecules 30, 5470-5475 (1997).

Rallison, J. M., "The deformation of small viscous drops and bubbles in shear flows," Annu. Rev. Fluid Mech. 16, 45-66 (1984).

Shapira, M., and S. Haber, "Low Reynolds number motion of a droplet in shear flow including wall effects," Int. J. Multiphase Flow 16, 305-321 (1990).

Shi, T. F., V. E. Ziegler, I. C. Welge, J. An, and B. A. Wolf, "Evolution of the interfacial tension between polydisperse "immiscible" polymers in the absence and in the presence of a compatibilizer," Macromolecules 37, 1591-1599 (2004).

Sibillo, V., G. Pasquariello, M. Simeone, V. Cristini, and S. Guido, "Drop deformation in microconfined shear 
flow," Phys. Rev. Lett. 97, 054502 (2006).

Sigillo, I., L. diSanta, S. Guido, and N. Grizzuti, "Comparative measurements of interfacial tension in a model polymer blend,” Polym. Eng. Sci. 37, 1540-1549 (1997).

Son, Y., N. S. Martys, J. G. Hagedorn, and K. B. Migler, "Suppression of capillary instability of a polymeric thread via parallel plate configuration," Macromolecules 36, 5825-5833 (2003).

Staben, M. E., A. Z. Zinchenko, and R. H. Davis, "Motion of a particle between two parallel plane walls in low-Reynolds-number Poiseuille flow," Phys. Fluids 15, 1711-1733 (2003).

Stone, H. A., "Dynamics of drop deformation and breakup in viscous flows," Annu. Rev. Fluid Mech. 26, 65-102 (1994).

Stone, H. A., A. D. Stroock, and A. Ajdari, "Engineering flows in small devices: Microfluidics toward a lab-on-a-chip," Annu. Rev. Fluid Mech. 36, 381-411 (2004).

Taylor, G. I., "The viscosity of a fluid containing small drops of another fluid," Proc. R. Soc. London, Ser. A 138, 41-48 (1932).

Taylor, G. I., "The formation of emulsions in definable flow fields," Proc. R. Soc. London, Ser. A 146, 501-523 (1934).

Tomotika, S., "On the instability of a cylindrical thread of a viscous fluid surrounded by another viscous fluid," Proc. R. Soc. London, Ser. A 146, 322-337 (1935).

Torza, S., R. G. Cox, and S. G. Mason, "Particle motions in sheared suspensions XXVII. Transient and steady deformation and burst of liquid drops," J. Colloid Interface Sci. 38, 395-411 (1972).

Tucker, C. L., III, and P. Moldenaers, "Microstructural evolution in polymer blends," Annu. Rev. Fluid Mech. 34, 177-210 (2002).

Tufano, C., G. W. M. Peters, P. D. Anderson, and H. E. H. Meijer, "Transient interfacial tension of partially miscible polymers," J. Colloid Interface Sci. 325, 130-140 (2008).

Tufano, C., G. W. M. Peters, H. E. H. Meijer, and P. D. Anderson, "Effects of partial miscibility on drop-wall and drop-drop interactions," J. Rheol. 54, 159-183 (2010).

Vananroye, A., R. Cardinaels, P. Van Puyvelde, and P. Moldenaers, "Effect of confinement and viscosity ratio on the dynamics of single droplets during transient shear flow," J. Rheol. 52, 1459-1475 (2008a).

Vananroye, A., P. J. A. Janssen, P. D. Anderson, P. C. J. Van Puyvelde, and P. Moldenaers, "Microconfined equiviscous droplet deformation: comparison of experimental and numerical results," Phys. Fluids 20, 013101 (2008b).

Vananroye, A., P. Van Puyvelde, and P. Moldenaers, "Effect of confinement on droplet breakup in sheared emulsions," Langmuir 22, 3972-3974 (2006).

Van Puyvelde, P., A. Vananroye, R. Cardinaels, and P. Moldenaers, "Review on morphology development of immiscible blends in confined flows," Polymer 49, 5363-5372 (2008).

Vinckier, I., P. Moldenaers, and J. Mewis, "Relationship between rheology and morphology of model blends in steady shear flow,” J. Rheol. 40, 613-631 (1996).

Young, Y.-N., J. Blawzdziewicz, V. Cristini, and R. H. Goodman, "Hysteretic and chaotic dynamics of viscous drops in creeping flows with rotation,” J. Fluid Mech. 607, 209-234 (2008).

Zhao, X., "Drop breakup in dilute Newtonian emulsions in simple shear flow: New drop breakup mechanisms," J. Rheol. 51, 367-392 (2007). 